\title{
Processor Core Model for Quantum Computing
}

\author{
Man-Hong Yung, ${ }^{1, *}$ Simon C. Benjamin, ${ }^{2,3}$ and Sougato Bose ${ }^{4}$ \\ ${ }^{1}$ Physics Department, University of Illinois at Urbana-Champaign, Urbana, Illinois 61801-3080, USA \\ ${ }^{2}$ Department of Materials, Oxford University, Oxford OX1 3PH, United Kingdom \\ ${ }^{3}$ Centre for Quantum Computation, Department of Physics, Oxford University, Oxford OX1 3PU, United Kingdom \\ ${ }^{4}$ Department of Physics and Astronomy, University College London, Gower Street, London WC1E 6BT, United Kingdom
}

(Received 2 September 2005; revised manuscript received 9 April 2006; published 5 June 2006)

\begin{abstract}
We describe an architecture based on a processing "core," where multiple qubits interact perpetually, and a separate "store," where qubits exist in isolation. Computation consists of single qubit operations, swaps between the store and the core, and free evolution of the core. This enables computation using physical systems where the entangling interactions are "always on." Alternatively, for switchable systems, our model constitutes a prescription for optimizing many-qubit gates. We discuss implementations of the quantum Fourier transform, Hamiltonian simulation, and quantum error correction.
\end{abstract}

DOI: 10.1103/PhysRevLett.96.220501

PACS numbers: 03.67.Lx

Typically, schemes for solid state quantum computing involve an array of qubits with some form of direct physical interaction coupling nearby elements [1-4]. In order to implement a specific algorithm, the major potential problem is that these schemes require the experimentalist to dynamically control the magnitude of each qubit-qubit interaction-effectively, to be able to switch it "on" and "off" precisely. A common idea for achieving this is to somehow dynamically manipulate the wave function overlap between a pair of neighboring qubits, while other nearby qubits are decoupled. This appears feasible but highly challenging. Moreover, even if a switching mechanism can be implemented, frequent switching is likely to increase the rate of decoherence. A deeper objection is that, by having the majority of a system's interactions off at a given moment, we are failing to maximally exploit its computational potential.

Recently, ideas have emerged [5,6] for computation in systems where the interaction remains always on. However, these proposals find ways to effectively pacify an interaction, and, therefore, one can make the same objection that they are not exploiting the full entangling power of the device. One class of system that does make full use of a set of permanent interactions is the mirrorinversion chain [7-12]. A chain of spins, with suitably engineered coupling strengths, has the property that a qubit placed on one end will later manifest at the other-even though at intervening times it is distributed over the chain. When more than one qubit is placed on the chain, each will manifest at the complementary site-but typically the qubits will have aquired an entangling phase. It has been observed [10] that this phase could, in principle, be employed to create certain classes of entangled state, graph states, which are the resource for one-way computation.

In this Letter, we demonstrate the potential of such engineered spin chains to directly implement arbitrary controlled multiqubit gates. The chain then acts as the computation core of our computer (see Fig. 1) - we need only supplement its free evolution with swap operations and single qubit manipulations. Note that this model is profoundly distinct from schemes involving a single qubit bus, e.g., the original ion trap schemes, since there the common mode represents only one qubit of information. We show that a controlled multiqubit gate can be constructed with exactly four free evolutions of the spin chain, independent of the number of spins involved. The controlling qubit can be any member of the spin chain, and the conditional unitary operations applied to the target qubits can be of any type. Such a gate can significantly reduce the number of elementary operations for quantum algorithms involving many nonlocal two-qubit operations.

We start with a finite chain of $N$ spin- $\frac{1}{2}$ particles confined within local potentials and interaction with their nearest neighbors. The Hamiltonian considered is

$$
H=\frac{1}{2} \sum_{j=1}^{N-1} \omega_{j}\left(\sigma_{j}^{x} \sigma_{j+1}^{x}+\sigma_{j}^{y} \sigma_{j+1}^{y}\right)+\frac{1}{2} \sum_{j=1}^{N} \lambda_{j}\left(\sigma_{j}^{z}+1\right),
$$

where the coupling constants $\left\{\omega_{j}, \lambda_{j}\right\}$ are real and, in general, distinct. We will adopt the convention that $\left|0_{j}\right\rangle$ $\left(\left|1_{j}\right\rangle\right)$ refers to be the spin-down state $|\downarrow\rangle(|\uparrow\rangle)$ at the site $j$.

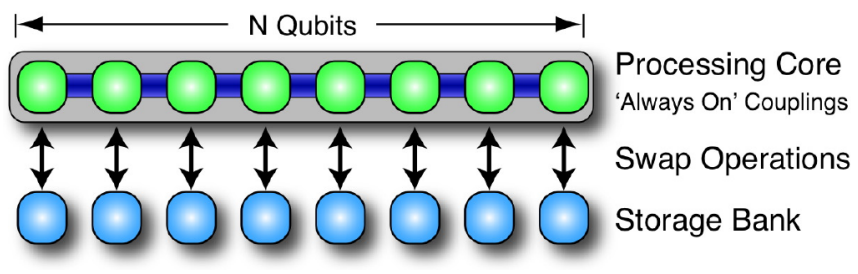

FIG. 1 (color online). Architecture of the processor-core model. The core is an engineered spin- $1 / 2$ chain with alwayson interactions. The storage bank consists of isolated sites where qubits can be swapped to and from the corresponding sites in the processing core. Controlled multitarget gates are constructed by the free evolution of the spin chain. 
This $X Y$ Hamiltonian is actually a very generic one describing the hopping process and can be manifested in many physical scenarios such as quantum dots coupled with cavity QED [2], nuclear spins coupled by a 2D electron gas [3], optical lattices [10], and Josephson junctions [4].

Next, we require that the core possess the so-called "mirror symmetry" [8]: $\omega_{j}=\omega_{N-j}$ and $\lambda_{j}=\lambda_{\bar{j}}$, where $\bar{j} \equiv N-j+1$ denotes the mirror-conjugate site of $j$. Let $|s\rangle \equiv\left|s_{1} s_{2}, \ldots, s_{N}\right\rangle, s_{j}=\{0,1\}$, be a particular spin configuration. Mirror inversion is said to occur when the state $|s\rangle$ is driven by the evolution operator $U(\tau)=e^{-i H \tau}$, for some fixed time period $\tau$, to the inverted state $|\bar{s}\rangle \equiv$ $\left|s_{N}, \ldots, s_{2} s_{1}\right\rangle$ (up to a phase factor). Note that the term mirror inversion refers to the inversion of classical states $|s\rangle$ in which all the sites have definite spin values. For a quantum state, being a superposition of the basis states in general, some internal phases will be acquired. However, it is exactly these entangling phases which we will exploit for gate construction.

It is shown [11] that the sufficient and necessary condition for mirror inversion in mirror symmetrical chains is determined by the eigenvalue spectrum of $H_{S}(\hbar=1)$, the single excitation subspace of $H$,

$$
e^{-i E_{k} \tau}=(-1)^{k} e^{-i \phi_{N}},
$$

where $E_{k}, k=0,1,2, \ldots, N-1$, is the $(k+1)$ th eigenvalue of $H_{S}$ and $\phi_{N}$ is some global phase independent of $k$. Because of the mirror symmetry, the coupling constants $\left\{\omega_{j}, \lambda_{j}\right\}$ can be determined by the eigenvalue spectrum. It is, therefore, an inverse eigenvalue problem [13]. Recently, many spectra $[7,8,12]$ satisfying the condition in Eq. (2) have been proposed. However, to keep our model general, we will continue our discussion without reference to any specific type of spectrum.

To construct multiqubit gates, we need to know the matrix elements of the evolution operator $U(\tau)$ in the $|s\rangle$ basis. Let $U \equiv U(\tau)$. By mapping our picture of localized spins to that of spinless fermions $[8,11]$, one can show that

$$
\mathrm{U}|s\rangle=e^{-n i \phi_{N}(-1)^{(n-m) / 2}}|\bar{s}\rangle,
$$

where $n$ is the number of spin-up states in $|s\rangle$, and $m=$ 0 (1) if $n$ is even (odd). The factor $(-1)^{(n-m) / 2}$ could be understood intuitively as follows: If $n$ is even (i.e., $m=0$ ), then the operation of mirror inversion (reordering the state) is equivalent to swapping $n / 2$ pairs of fermions and, similarly, for odd $n$, except the factor should be the same as that of $n-1$ fermions. The phase factor $e^{-n i \phi_{N}}$ is a consequence of Eq. (2). In fact, the phase $\phi_{N}$ can be set to zero if an appropriate spectrum of $H_{S}$ is chosen. In this case, it has been demonstrated [10] that the operator $U(\tau)$ alone can generate a fully connected graph state. However, in constructing multiqubit gates, spurious correlations among qubits in the graph state have to be eliminated. This can be achieved with the help of an ancilla qubit within the storage array, initialized to be $|0\rangle_{a}$. Let $\mathrm{S}_{x}$ represent the swap operation between the spin at the site $\bar{x}$ (complement of $x$ ) and the ancilla. We apply $\mathrm{S}_{x}$ to the state in Eq. (3) and allow the engineered chain to evolve once more, i.e., applying $\mathbf{Z}^{x} \equiv \mathrm{US}_{x} \mathrm{U}[14]$ to the initial state $|0\rangle_{a} \otimes|s\rangle$. Then from (3) the final state is

$$
e^{-\left(2 n-s_{x}\right) i \phi_{N}}(-1)^{s_{x}(n-1)}\left|s_{x}\right\rangle_{a} \otimes\left|s_{1} s_{2} \ldots 0_{x} \ldots s_{N}\right\rangle,
$$

which is the same for both odd and even $n$. Here we have only assumed a swap operation performed between the site $\bar{x}$ and the ancilla. Therefore, the qubit staying at the ancilla spin cannot be transferred back to the spin chain at this stage. However, as we shall see [cf. Eq. (7)], a more general multiqubit gate can be constructed based on $Z^{x}$ and all of the qubits can reside in their original locations at the end of the operation.

The next step is to interpret the result (4) in terms of the quantum circuit model. The phase factor $e^{-\left(2 n-s_{x}\right) i \phi_{N}}$ can be regarded as a result of $N$ local phase gates $\mathrm{R}_{j}\left(-2 \phi_{N}\right)$, where $\mathrm{R}_{j}(\varphi) \equiv\left|0_{j}\right\rangle\left\langle 0_{j}\left|+e^{i \varphi}\right| 1_{j}\right\rangle\left\langle 1_{j}\right|$, acting on all qubits, and one extra phase gate $\mathrm{R}_{j}\left(\phi_{N}\right)$, acting on the spin at site $x$ alone. On the other hand, the factor $(-1)^{s_{x}(n-1)}$ can be considered as due to the application of controlled- $\sigma_{z}$ to all qubits, except the spin at site $x$ which is encoded with the controlling qubit. Suppose we now apply local operations to get rid of all the controlled phase gates $\mathrm{R}_{j}$ (or simply choose an eigenvalue spectrum such that $\phi_{N}=0$ ); effectively, we have constructed a controlled multitarget gate, which requires two free evolutions of the engineered Hamiltonian for any $N$. Note that, for this multiqubit gate generated by $\mathbf{Z}^{x}$, the $\sigma_{z}$ gate has to be applied to all qubits, controlled by a single qubit at site $\bar{x}$. However, the $\sigma_{z}$ gate can be converted into controlled $-\mathrm{V}_{j}[15]$, where

$$
\mathrm{V}_{j}=\left(\begin{array}{cc}
\sin \theta_{j} & e^{i \varphi_{j}} \cos \theta_{j} \\
e^{-i \varphi_{j}} \cos \theta_{j} & -\sin \theta_{j}
\end{array}\right),
$$

through local operations $A_{j}$ provided that the relations $\mathrm{A}_{j} \sigma_{z} \mathrm{~A}_{j}^{\dagger}=\mathrm{V}_{j}$ and $\mathrm{A}_{j} \mathrm{~A}_{j}^{\dagger}=\mathrm{I}_{j}$, where $\mathrm{I}_{j}$ is the identity operator, are satisfied. Operationally, we denote the construction of this controlled multitarget gate by $\mathrm{V}^{x} \equiv$ $A Z^{x} A^{\dagger}$, where $A \equiv \prod_{j=1}^{N} A_{j}$ (and similarly for $A^{\dagger}$ ). An immediate application of this gate is that, if we initialize the controlling qubit to be $|0\rangle+|1\rangle$ and the rest $|000, \ldots, 0\rangle$, it can efficiently generate a cat state $|000, \ldots, 0\rangle+|111, \ldots, 1\rangle$, which is interesting for various applications including single qubit measurement and encoding error correcting codes, such as the Shor's code.

The controlled operations $\mathrm{V}_{j}$ are not yet completely general: For example, the phase gate $\mathrm{R}_{j}(\varphi)$ and the identity operator $I_{j}$ are excluded. We can construct a more general controlled multitarget gate, which applies arbitrary unitary operations $\mathbf{W}_{j}$ on the qubits. To proceed, consider applying $\mathrm{U}$ to the state $\left|s_{1} s_{2}, \ldots, 0_{x}, \ldots, s_{N}\right\rangle$, which is assumed to contain $n$ spin-up states. The phase factors generated are 
exactly the same as that in Eq. (3). Now we can apply $\mathrm{US}_{x}$ to the resulting state; $\mathrm{S}_{x}$ returns $\left|s_{x}\right\rangle_{a}$ from the ancilla to the core. The final state is $e^{-\left(2 n+s_{x}\right) i \phi_{N}}(-1)^{s_{x} n}|s\rangle$, which can also be considered as an controlled multitarget gate analogous to the one generated by $Z^{x}|s\rangle$. For simplicity, we again assume $\phi_{N}=0$. To construct a more general unitary matrix

$$
\mathrm{W}_{j}=\left(\begin{array}{cc}
e^{i\left(\alpha_{j}-\beta_{j}-\delta_{j}\right)} \cos \gamma_{j} & -e^{i\left(\alpha_{j}-\beta_{j}+\delta_{j}\right)} \sin \gamma_{j} \\
e^{i\left(\alpha_{j}+\beta_{j}-\delta_{j}\right)} \sin \gamma_{j} & e^{i\left(\alpha_{j}+\beta_{j}+\delta_{j}\right)} \cos \gamma_{j}
\end{array}\right),
$$

including the identity operator, one can always choose a set of local operations $\mathrm{A}_{j}, \mathrm{~B}_{j}$, and $\mathrm{C}_{j}$ such that the relations $\mathrm{A}_{j} \mathrm{~B}_{j} \mathrm{C}_{j}=\mathrm{I}_{j}$ and $e^{i \alpha_{j}} \mathrm{~A}_{j} \mathrm{ZB}_{j} \mathrm{ZC}_{j}=\mathrm{W}_{j}$ [15] are satisfied. Then, the controlled- $W_{j}$ gate $\mathrm{W}^{x}$, with the controlling qubit at site $x$, can be constructed by the following sequence of operations: $W^{x}=A Z^{x} B Z^{x} C$, where $A, B$, and $C$ are the tensor products of local operators $\mathrm{A}_{j}, \mathrm{~B}_{j}$, and $\mathrm{C}_{j}$, respectively. Equivalently,

$$
\mathbf{W}^{x}=\left|0_{x}\right\rangle\left\langle 0_{x}|\otimes \mathbf{I}+| 1_{x}\right\rangle\left\langle 1_{x}\right| \otimes \prod_{j \neq x} \mathbf{W}_{j} .
$$

The circuit diagram for an example $W^{1}$ is shown in Fig. 2. For any $N$, the cost of generating $\mathbf{W}^{x}$ includes four free evolutions of the engineered chain, two swap operations with the same ancilla and local operations.

We now describe some example applications of this model. For comparison, we also consider a fully switched system, having a Hamiltonian similar to (1) but with $\omega_{j}$ dynamically switched so as to couple only pairs of qubits simultaneously, although it may do so in parallel-i.e., $\omega_{i} \omega_{i+1}=0$ at all times. A real fully switched system could, presumably, activate several adjacent interactions: Our results here can be seen as a prescription for doing precisely that. The primary gain in efficiency will, of course, be a reduction in the number of switching events - but, remarkably, there can also be an absolute speedup by a fixed factor, as we presently discuss.

One of the immediate applications of the controlled multitarget gate $\mathbf{W}^{x}$ is the operation of quantum Fourier transform $(\mathrm{QFT})$, which is a key ingredient in many quan-

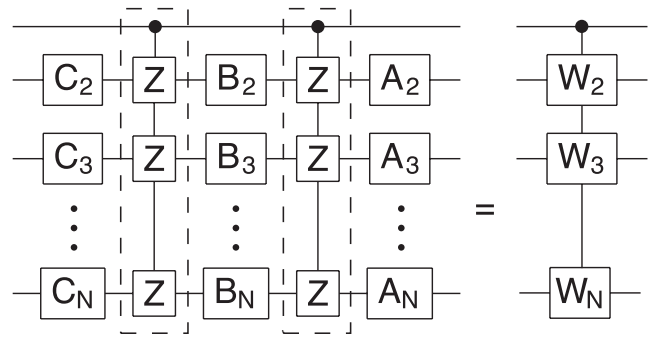

FIG. 2. The circuit diagram of a controlled multitarget gate $W^{1}=A Z^{1} B Z^{1} C$ constructed by the free evolution of an engineered spin chain core. The parts inside the dashed boxes are $Z^{1}$, which involves two free evolutions of the spin chain and one ancilla in the store. tum algorithms such as the Shor's algorithm. In the above notations, the standard QFT circuit can be constructed by applying the multiqubit gates and the Hadamard gates alternatively,

$$
\mathrm{QFT}=\mathrm{H}_{N} \mathrm{~W}^{N-1} \mathrm{H}_{N-1} \cdots \mathrm{W}^{2} \mathrm{H}_{2} \mathrm{~W}^{1} \mathrm{H}_{1},
$$

where $\mathrm{W}_{j}=\mathrm{R}_{j}\left(\pi / 2^{j-x}\right)$ for $j>x$ and $\mathrm{W}_{j}=\mathrm{I}_{j}$ otherwise. Here each joint operation $W_{j}$ costs exactly four free evolutions, including two swaps. The QFT circuit depth is, therefore, $O(N)$. For the switched model, the circuit depth is also $O(N)$, but the absolute number of switching events is $O\left(N^{2}\right)$.

The second application is the simulation of the evolution of an "artificial" Hamiltonian $H_{A}$ formally representing a joint interaction between $r$ spin-1/2 particles,

$$
H_{A}=\sigma_{1}^{z} \otimes \sigma_{2}^{z} \otimes \sigma_{3}^{z} \cdots \otimes \sigma_{r}^{z},
$$

which is locally equivalent to the class of the Hamiltonian of the form $\sigma_{1}^{w_{1}} \otimes \sigma_{2}^{w_{2}} \otimes \sigma_{3}^{w_{3}} \cdots \otimes \sigma_{r}^{w_{r}}$, where $\sigma_{j}^{w_{j}}=\sigma_{j}^{x}$, $\sigma_{j}^{y}$, or $\sigma_{j}^{z}$. Although it is unlikely to find a group of spin- $1 / 2$ particles interacting naturally under the Hamiltonian $H_{A}$, some higher dimensional systems can be mapped by these two-level systems. Moreover, the form of $H_{A}$ can be considered as a basic building block for simulating more complex Hamiltonians through the short-time approximation: $e^{i(A+B) \Delta t}=e^{i A \Delta t} e^{i B \Delta t}+O\left(\Delta t^{2}\right)$.

Consider an engineered core of $N+1$ spins, with $\phi_{N}=$ 0 , initialized as $|0 s\rangle \equiv\left|0 s_{1} s_{2}, \ldots, s_{N}\right\rangle$. The quantum circuit for simulating the evolution operator $U_{A}(\Delta t)=$ $e^{-i H_{A} \Delta t}$ for (9) can be constructed by the following sequence of operations [15]:

$$
U_{A}(\Delta t)=\mathrm{H}_{0} \mathrm{~W}^{0} \mathrm{H}_{0} \mathrm{~T}_{0}(\Delta t) \mathrm{H}_{0} \mathrm{~W}^{0} \mathrm{H}_{0},
$$

where $\mathrm{T}_{0}(\Delta t)=\exp \left(-i \sigma_{0}^{z} \Delta t\right)$ and $W_{j}=\sigma_{j}^{z}$ (or $W_{j}=I_{j}$, if the qubit at site $j$ is not involved). The basic idea of this construction is to store the parity (i.e., $m=\{0,1\}$ ) of the sites $j=1,2,3, \ldots, N$ to site 0 . The phase generated by the local operation $\mathrm{T}_{0}$, depending on the parity, is exactly the one required for $H_{A}$. From (7), it is apparent that the series of nonlocal operations $W^{0}$ can be achieved by four free evolutions of the engineered chain of $N \geq r$ spins. This scheme offers the flexibility of generating interactions involving a various number of spins using the same spin chain. The costs of generating each type of interaction are fixed (eight free evolutions). Alternatively, if one just needs to generate interactions with a fixed number of qubits, i.e., $r=N$, the cost can be reduced to two free evolutions and no ancilla is needed. The sequence of operations in this case is

$$
U_{A}(\Delta t)=\mathrm{H}_{0} \mathrm{UH}_{\overline{0}} \mathrm{~T}_{\overline{0}}(\Delta t) \mathrm{H}_{\overline{0}} \mathrm{UH}_{0} .
$$

The crucial observation for obtaining (11) is that $\mathrm{H}_{\overline{0}} \mathrm{UH}_{0}|0 s\rangle=(-1)^{(n-m) / 2}|\bar{s} m\rangle$. Thus, the desired phase can be obtained by applying the local operator $\mathrm{T}_{\overline{0}}$ at site $\overline{0}$. 
The processor-core model will also be advantageous in running quantum algorithms in a fault tolerant fashion with concatenated code words. For example, in the Steane code, six gates of the class $\mathbf{W}^{x}$ are required for error syndrome measurement (Fig. 10.16 in Ref. [15]). In our approach, each level of concatenation just multiplies the number of targets in each $Z^{x}$ by 7 [16] but does not increase the number of applications of such gates. However, the number of elementary switching operations required by a fully switched system to realize $\mathrm{W}^{x}$ increases sevenfold with each level of concatenation.

The discussions above highlight potential gains in terms of simplicity: the circuit depth or total number of switching operations. It is also interesting to ask: Can the total time be reduced by applying the processor-core model? We can quickly conclude that any speedup must be bounded, since the fundamental operation $U$ [Eq. (3)] can be simulated on a fully switched array in time $O(N)$ [17], while $U$ also takes time $O(N)$ to evolve on our processor core (given a fixed maximum interaction strength). Interestingly, there can be speedups within by bounded factors. To make a definite statement, we specialize to a core with a linear spectrum, i.e., $\Delta_{k}=E_{k}-E_{k+1}$, being constant (e.g., $[7,8,12])$ since this is the time-optimal choice for a given spectral range [18]. Let us compare the time required for a simple state transfer, i.e., $|100 \ldots 0\rangle \rightarrow|0 \ldots 001\rangle$. Given that the maximum interaction strength $\omega_{\max } \simeq N / 4$ (see Ref. [7]) scales as $N$, then the evolution time of the processor core is simply $\pi$ for all $N$. On the fully switched system, the time required for each swap is $\pi / 2 \omega_{j}$; thus, the total time required is $T(N)=\sum_{j=1}^{N-1}\left(\pi / 2 \omega_{j}\right)$. (For the fully switched system, each state transfer must be completed before the next is initiated.) One can easily show that $T(N) \geq(N-1) \pi / 2 \omega_{\max } \simeq 2 \pi(N-1) / N$. Thus, this always-on processing core can be superior by a factor of 2 for large $N$.

Finally, we remark that the periods of the free processorcore evolution can be relatively robust versus timing errors $\tau \rightarrow \tau+\delta t$ in subsequent swaps to the store. Consider the most general initial state $|\bar{\psi}\rangle=\sum_{j} \alpha_{j}\left|\bar{s}_{j}\right\rangle$, where $\sum_{j}\left|\alpha_{j}\right|^{2}=1$. If the evolution time is taken perfectly, we expect the final state to be $|\psi\rangle=\sum_{j} \alpha_{j} e^{i \phi_{j}}\left|s_{j}\right\rangle$, where $\phi_{j}$ represents the overall phase in Eq. (3) for the spin configuration $\left|s_{j}\right\rangle$. If not, we have $\langle\psi|U(\tau+\delta t)| \bar{\psi}\rangle=\sum_{j, k} \alpha_{j}^{*} \alpha_{k} \times$ $e^{i\left(\phi_{k}-\phi_{j}\right)}\left\langle s_{j}|U(\delta t)| s_{k}\right\rangle \equiv 1+i A \delta t+B \delta t^{2}+O\left(\delta t^{3}\right)$. Here both $A$ and $B$ are real. The error $\epsilon \equiv 1-\mid\langle\psi| U(\tau+$ $\delta t)\left.|\bar{\psi}\rangle\right|^{2}$ is, therefore, just second order in $\delta t$.

In conclusion, we have demonstrated how to construct controlled multitarget gates through the natural evolution of a processor core where interactions are always on. This model allows computation with physical systems where the entangling interactions are not switchable. Alternatively, in switchable systems, our protocol can play an important role in simplifying multiqubit operations. We demonstrated this by showing that the fully switched model is fundamentally more complex for certain important algorithmic tasks. For various operations spanning many qubits, including long range state transfer, the temporal requirement for the processor-core model is less than or equal to that of the fully switched model. Therefore, in terms of the reduction of dynamical control, many schemes presently described in terms of two-qubit gates can be enhanced by incorporating the processor-core concept.

M. H. Y. acknowledges the support of the Croucher Foundation and the QIPIRC. S.C.B. is supported by the Royal Society.

Note added in proof. - Recently, two interesting papers have emerged that offer new ways to perform mirror inversion, thus potentially further broadening the range of systems that can implement the techniques we introduce here $[19,20]$.

*Electronic address: myung2@uiuc.edu

[1] An archetypal example is provided by B. E. Kane, Nature (London) 393, 133 (1998).

[2] A. Imamoglu et al., Phys. Rev. Lett. 83, 4204 (1999).

[3] D. Mozyrsky et al., Phys. Rev. Lett. 86, 5112 (2001).

[4] A. M. Zagoskin et al., cond-mat/0603753.

[5] X. Zhou et al., Phys. Rev. Lett. 89, 197903 (2002).

[6] S. C. Benjamin and S. Bose, Phys. Rev. Lett. 90, 247901 (2003).

[7] M. Christandl et al., Phys. Rev. Lett. 92, 187902 (2004).

[8] C. Albanese et al., Phys. Rev. Lett. 93, 230502 (2004).

[9] P. Karbach and J. Stolze, Phys. Rev. A 72, 030301(R) (2005).

[10] S. R. Clark et al., New J. Phys. 7, 124 (2005).

[11] M.-H. Yung and S. Bose, Phys. Rev. A 71, 032310 (2005).

[12] T. Shi et al., Phys. Rev. A 71, 032309 (2005).

[13] B. N. Parlett, The Symmetric Eigenvalue Problem (Society for Industrial and Applied Mathematics, Philadelphia, 1998).

[14] Subscripts denote the site to be acted upon, and superscripts denote the site of the controlling qubit.

[15] M. A. Nielsen and I. L. Chuang, Quantum Computation and Quantum Information (Cambridge University Press, Cambridge, England, 2000).

[16] Each level of concatenation simply replaces a single $Z$ operator by $Z_{1} Z_{2}, \ldots, Z_{7}$.

[17] One simply alternates between swaps on all pairs $\left(s_{2 i}, s_{2 i+1}\right)$ and then all pairs $\left(s_{2 i-1}, s_{2 i}\right)$. Each qubit will, at some point, be adjacent to every other; thus, by interleaving nearest-neighbor phase gates with the swap operations, we implement the correct phase, still within $O(N)$ time steps. The total number of gates is $O\left(N^{2}\right)$.

[18] From Eq. (2), $\Delta_{k} \tau \geq \pi$ for all $k$. To compare different eigenvalue spectra, we require them to be normalized as $E_{\max }-E_{\min }=1$. The minimal evolution time of the linear spectrum is $\tau=(N-1) \pi$. For any nonlinear spectrum, there must exist at least one $\Delta_{k}<1 /(N-1)$, and, hence, $\tau>(N-1) \pi$.

[19] R. Raussendorf, Phys. Rev. A 72, 052301 (2005).

[20] J. Fitzsimons and J. Twamley, quant-ph/0601120. 